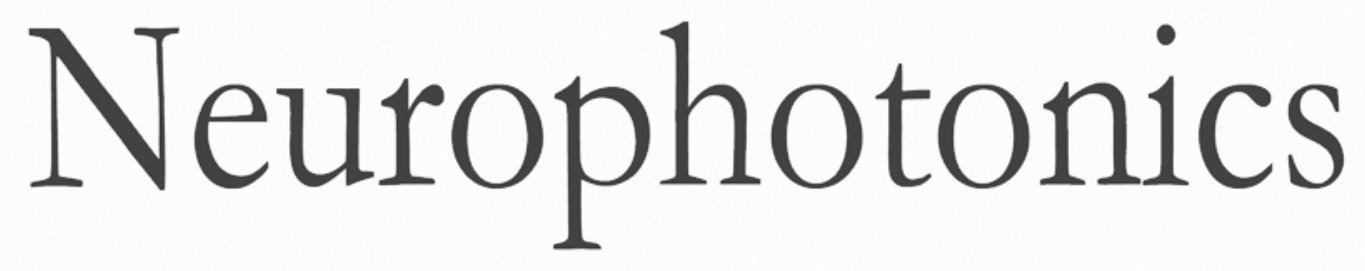

\title{
Review of functional near-infrared spectroscopy in neurorehabilitation
}

Masahito Mihara

Ichiro Miyai 


\title{
Review of functional near-infrared spectroscopy in neurorehabilitation
}

\author{
Masahito Mihara ${ }^{a, b, *}$ and Ichiro Miyai ${ }^{c}$ \\ aOsaka University, Graduate School of Medicine, Department of Neurology, 2-2 Yamadaoka, Suita, Osaka 565-0871, Japan \\ bosaka University, Global Center for Medical Engineering and Informatics, Division of Clinical Neuroengineering, 2-2 Yamadaoka, \\ Suita, Osaka 565-0871, Japan \\ 'Morinomiya Hospital, Neurorehabilitation Research Institute, 2-1-88 Morinomiya, Jyoto-ku, Osaka, Osaka 536-0025, Japan
}

\begin{abstract}
We provide a brief overview of the research and clinical applications of near-infrared spectroscopy (NIRS) in the neurorehabilitation field. NIRS has several potential advantages and shortcomings as a neuroimaging tool and is suitable for research application in the rehabilitation field. As one of the main applications of NIRS, we discuss its application as a monitoring tool, including investigating the neural mechanism of functional recovery after brain damage and investigating the neural mechanisms for controlling bipedal locomotion and postural balance in humans. In addition to being a monitoring tool, advances in signal processing techniques allow us to use NIRS as a therapeutic tool in this field. With a brief summary of recent studies investigating the clinical application of NIRS using motor imagery task, we discuss the possible clinical usage of NIRS in braincomputer interface and neurofeedback. ๑ The Authors. Published by SPIE under a Creative Commons Attribution 3.0 Unported License. Distribution or reproduction of this work in whole or in part requires full attribution of the original publication, including its DOI. [DOI: 10.1117/1.NPh.3.3 $.031414]$
\end{abstract}

Keywords: near-infrared spectroscopy; rehabilitation; neurofeedback; functional recovery; stroke.

Paper 16005SSVRR received Jan. 16, 2016; accepted for publication Jun. 21, 2016; published online Jul. 12, 2016.

\section{Introduction}

Near-infrared spectroscopy (NIRS) is a unique noninvasive neuroimaging tool that has several potential advantages including less onerous constraints during measurement, relatively small and portable equipment, and its almost completely safe and noninvasive nature. Despite several shortcomings including difficulty in monitoring deep brain structures, relatively poor spatial resolution, and possible contamination with extra-brain artifacts, the NIRS system is suitable for clinical settings and has been introduced into the field of rehabilitation medicine. In this brief review, we focus on two major usages of the NIRS in the neurorehabilitation field.

One application of NIRS in neurorehabilitation is as a monitoring tool. Functional neuroimaging tools including functional magnetic resonance imaging (fMRI) and NIRS are used for investigating the mechanisms of functional recovery after brain injury. In patients with acute brain injury such as stroke, the time course of functional recovery assumes an exponential shape, with a faster recovery in the initial few weeks followed by a slower recovery in the next few months. ${ }^{1,2}$ It is believed that the faster recovery is dominantly achieved by focal processes including reduction of parenchymal edema or recanalization of blood flow, and that the slower recovery is made possible by the adaptive plasticity of the brain including unmasking or disinhibiting the potential aberrant neural network and vicariation of function. ${ }^{3,4}$

The second application of NIRS is as a therapeutic tool. Recently, there has been much interest in developing the braincomputer interface (BCI) technology to improve the activities of daily living (ADL) and to restore function in patients severely

*Address all correspondence to: Masahito Mihara, E-mail: mihara@ neurol.med .osaka-u.ac.jp affected by neurological diseases. ${ }^{5,6}$ Because of the noninvasive and portable nature of NIRS, many researchers have tried to develop functional NIRS-based BCI (see Naseer and Hong ${ }^{7}$ for a review). In addition, NIRS could be used for augmenting cortical plasticity. It has been suggested that cortical activity feedback to the subject using electroencephalography (EEG) might help to modulate cortical activity of the subject. ${ }^{8}$ For decades, this technique, known as "neurofeedback," has been applied in the treatment of attention deficiency and hyperactivity disorder ${ }^{9}$ and epilepsy. ${ }^{10}$

\section{Application of Near-Infrared Spectroscopy as a Monitoring and Investigation Tool in Neurorehabilitation}

\subsection{Upper Limb Function}

\subsubsection{Functional recovery after brain damage}

There is ample neuroimaging evidence, mainly obtained through fMRI studies, that functional reorganization plays an essential role in functional recovery after brain damage. ${ }^{3,11-13}$ As observed in an fMRI study, NIRS can also detect altered cortical perfusion and activation as a course of recovery after stroke (see Ref. 14 for review), and test-retest reliability has been confirmed to be relatively high. ${ }^{15}$ In comparison with fMRI, Kato et al. ${ }^{16}$ revealed that NIRS can be used to detect a similar cortical activation pattern, showing contralateral motor cortex dominant activation in the unaffected hand, and bilateral motor cortex activation in the affected hand in six patients with hemiplegia and five healthy subjects. In a case series that observed longitudinal changes in a movement task using the affected hand during 10 consecutive days of rehabilitative intervention of forced use of the affected limb [constraint-induced movement therapy (CIMT)], ${ }^{17}$ it was observed that gradual decrement of the 
bilateral cortical activation and interhemispheric balance of cortical activation shifted to the contralateral (affected) motor cortex. These findings were confirmed by a following group study with five subjects with subcortical stroke. ${ }^{18}$ In addition to patients with stroke, functional reorganization of the cerebral cortex by CIMT intervention including altered functional connectivity can be detected in patients with cerebral palsy. ${ }^{19}$

\subsubsection{Motor learning}

In addition to monitoring the functional reorganization after brain damage, NIRS can be used as a tool for investigating the neural underpinnings of motor learning. Motor learning is vital and essential in our daily life as well as during rehabilitation when patients have to relearn the usage of their paretic limbs. To date, most human neuroimaging studies regarding motor learning have used positron emission tomography (PET) or fMRI. Because of the methodological limitation of these techniques, most studies investigated the learning of finger or foot movement with subjects in a lying position. However, since in daily situations, most movements are executed while subjects are sitting or standing, motor learning usually occurs under the requirement of postural control. Because of lesser postural restrictions during measurement, NIRS is a suitable tool for investigating motor learning under daily circumstances.

Using functional NIRS, several researchers have investigated learning-related cortical activation change using gross upper limb movement. Leff et al. ${ }^{20}$ have shown larger prefrontal activation during learning of a surgical knotting procedure in a novice student than an expert consultant, suggesting prefrontal involvement for skill acquisition. Hatakenaka et al. ${ }^{21}$ also studied the change in cortical activation during the pursuit rotor (PR) task in healthy subjects and in poststroke patients with ataxia. ${ }^{22}$ In a PR task, subjects are asked to keep a stylus on a rotating target; it can be used to evaluate the skills for motor sequence learning. The PR task does not require precise control of finger movements, but it requires motor control of proximal parts of the upper extremity including the shoulder and elbow as well as postural control for sitting. ${ }^{23}$

In both healthy subjects and those with stroke, the PR task performance improved with repetitions, although only healthy subjects reached a plateau. The PR task improvement was significantly correlated with improvement of ADL in patients with stroke. During the PR task, cortical activation was observed in (b)

\section{(a)}

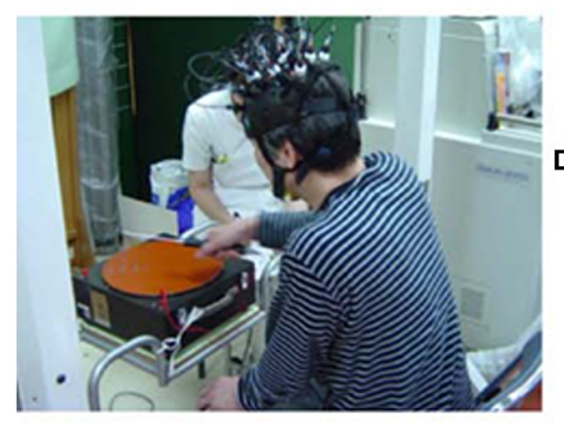

(c)

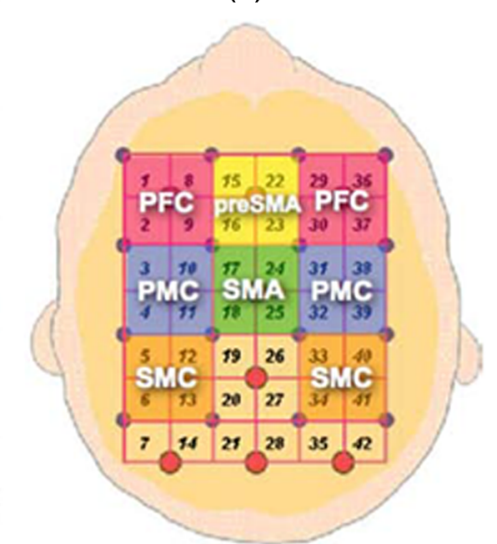

(d)

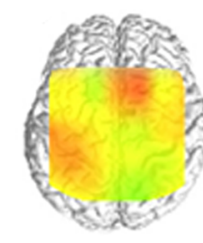

Cycle 1

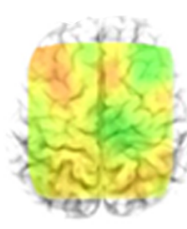

Cycle 1

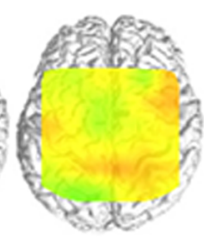

Cycle 2

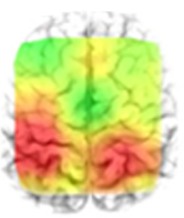

Cycle 2

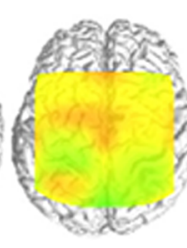

Cycle 3

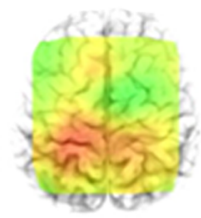

Cycle 3

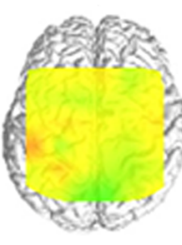

Cycle 4

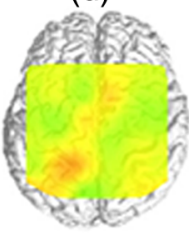

Cycle 5 (e)

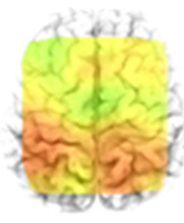

Cycle 4

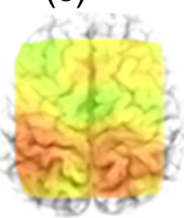

Cycle 5

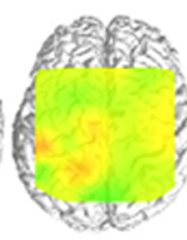

Cycle 6

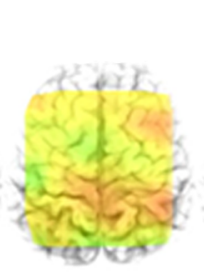

Cycle 6

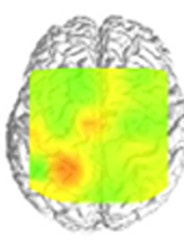

Cycle 7

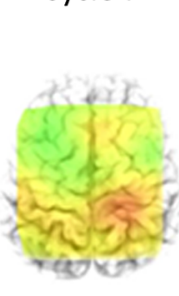

Cycle 7

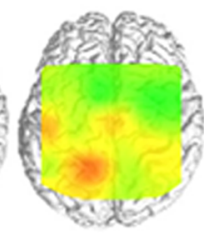

Cycle 8
oxyHb
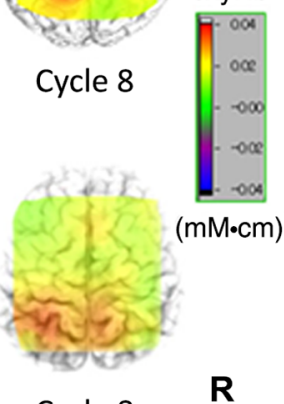

Cycle 8

Fig. 1 Motor learning study using NIRS: (a) experimental setting of the PR, (b) location of optodes, (c) covering cortical surface by each channel, (d) longitudinal cortical activation changes in a healthy subject, and (e) longitudinal cortical activation changes in a stoke patient with ataxia. Reproduced from the articles by Hatakenaka et al., ${ }^{21,22}$ where details are reported. PFC, prefrontal cortex; preSMA, presupplementary motor area; SMA, supplementary motor area; PMC, premotor cortex; and SMC, sensorimotor cortex. 
the sensorimotor, prefrontal, and premotor cortices (PMC). Interestingly, the center of cortical activation was shifted caudally from the presupplementary motor area (pre-SMA) to the supplementary motor area (SMA) with cycle repetitions in healthy subjects. In contrast, the presupplementary motor activation was sustained in patients with poststroke ataxia (Fig. 1). Their data also suggested that the caudal shift of the pre-SMA to the SMA significantly correlated with the PR task improvement in patients with stroke. These data suggested the different roles of the pre-SMA and SMA in the motor skill learning process.

\subsection{Gait and Balance Control}

\subsubsection{Neural mechanism for gait control}

Although habitual bipedal standing and locomotion in humans help to maximize the dexterity of hands for developing tools, the unstable nature of bipedal standing increases the tendency to fall, leading to severe injuries. In fact, one community-based study revealed that more than one third of people aged above 65 years fell at least once a year and that the proportion increased to $50 \%$ by the age of 80 years. ${ }^{24}$ Accumulated evidence from animal studies suggests that the subcortical structures contribute to gait and balance control. ${ }^{25,26}$ However, recent studies have suggested that the cerebral cortex is involved in unstable bipedal human balance control. ${ }^{27-29}$ For investigating the neural mechanism of postural control in humans, NIRS is the most suitable neuroimaging technique since it does not necessitate postural constraint as in fMRI or PET.

Using NIRS and a weight-balancer system for avoiding excessive motion artifact, we have reported gait-related cortical activation while walking on a treadmill, ${ }^{30}$ which showed symmetrical activation in the medial sensorimotor cortex (SMC) and SMA (Fig. 2). In addition, Suzuki et al. ${ }^{31}$ found that the prefrontal cortex (PFC) activates only during the acceleration phase of the locomotion and the activity decreases during steady locomotion. Interestingly, the prefrontal activation change was more prominent at the higher locomotor speed suggesting that the PFC is more involved in the adaptation to locomotion, and that the medial SMC is involved in stabilizing the gait.

Other studies have also suggested the prefrontal involvement for locomotor control. Holtzer et al. investigated the prefrontal activity during gait with and without a concurrent talking task. The prefrontal activation increased more in dual-task condition than normal gait in nondemented elderly subjects and larger prefrontal activation correlated with larger stride length and better cognitive performance. ${ }^{32}$ Al-Yahya et al. ${ }^{33}$ have also reported

(a)

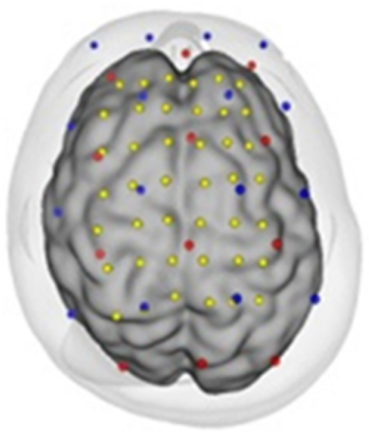

(b)

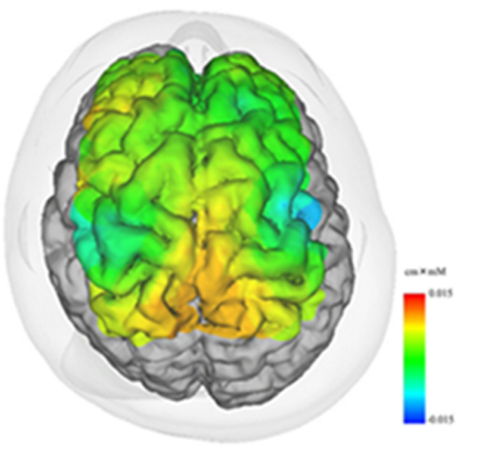

that the prefrontal activity was more prominent in dual-task walking compared with single-task walking in both healthy subjects and patients with stroke. These findings suggested that the PFC is involved in the allocation of attentional resources during dual-task walking situations. Interestingly, it is also reported that in patients with neuropathic gait disturbance, larger prefrontal activation is correlated with faster gait speed and worse cognitive performance, suggesting that the strategy for attentional allocation was different between patients and normal elderly. ${ }^{34}$

In patients with stroke with hemiparesis, gait-related cortical activation showed an asymmetric pattern in the SMC and intervention by therapists could alter cortical activation. ${ }^{35} \mathrm{~A}$ recent study revealed that a light haptic touch of an unaffected finger could facilitate cortical activation and diminish asymmetric cortical activation patterns during gait in patients with stroke. ${ }^{36}$ These findings may warrant conventional therapeutic intervention by therapists. A longitudinal study revealed that the motorrelated cortex activation in the affected hemisphere, especially in the PMC, increased with functional recovery, ${ }^{37}$ suggesting that the PMC plays a compensatory role in poststroke gait recovery, consistent with previous findings. ${ }^{38}$ Functional reorganization for lower limb function after stroke was also suggested by a study using cycling task. Lin et al. investigated cortical activation during active and passive cycling task, and they found that cortical activation in the unaffected PMC was higher and cycling performance was better in active cycling condition with speed feedback. These findings suggested that the unaffected premotor activation may play a compensative role in stroke recovery. ${ }^{39}$

However, the pattern of functional reorganization is not uniform and may vary with the type of lesion or symptom of each patient. In patients with stroke with ataxia, gait-related activation in the medial SMC is comparable to that of healthy subjects. However, the prefrontal activation was sustained even in the steady gait condition throughout the walking phase ${ }^{40}$ (Fig. 3). Increased prefrontal activation was also found in patients with chronic degenerative ataxia, Caliandro et al. ${ }^{41,42}$ reported a significant positive correlation between cortical activation in the PFC and step width. These findings suggested that the PFC might compensate for the increased variability of limb movements in patients with ataxia.

\subsubsection{Neural mechanism for postural control}

Using functional NIRS, we reported cortical activation associated with postural adjustment after predictable or unpredictable external postural perturbation. ${ }^{43}$ A significant task-related

Fig. 2 Cortical activation during gait in a healthy subject: (a) optode location and estimated cortical projection, (b) cortical activation map during gait, and (c) experimental setting for a locomotor task. 


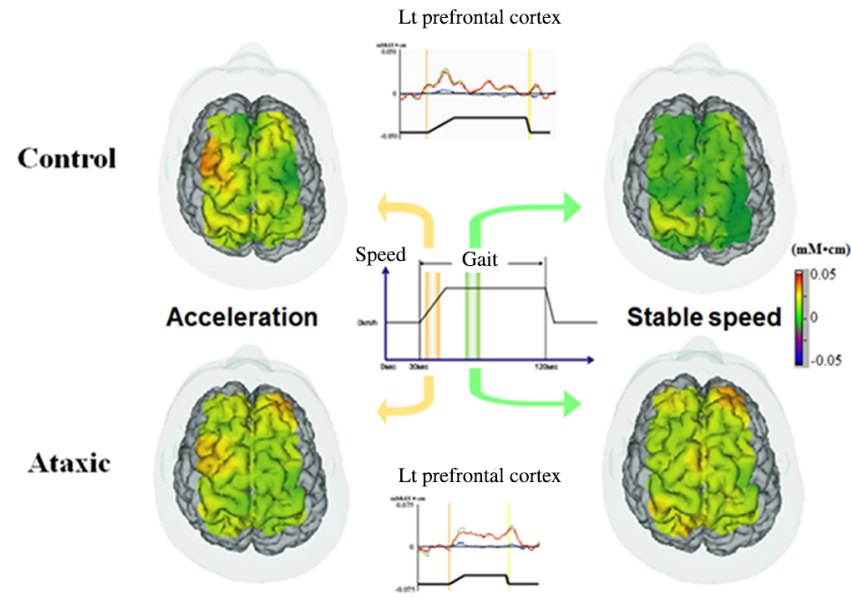

Fig. 3 Gait-related cortical activation in healthy subjects and patients with ataxic stroke. Reproduced from the article by Mihara et al.,40 where details are reported. (c) 2012 Masahito Mihara. Adapted from Applications of near infrared spectroscopy in neurorehabilitation. In Infrared Spectroscopy/Book 1 (ISBN: 979-953-307-362-9); originally published under CC BY 3.0 license. Available from doi: 10.5772/2655.

OxyHb increase in the bilateral PFC was observed regardless of preceding warning cues, and the SMA and the parietal association cortex were activated only in the predictable condition (Fig. 4). The prefrontal involvement in the active balance tasks was confirmed using a virtual tilt board task, which showed a positive correlation between task difficulty and prefrontal activation. ${ }^{44}$ In addition, Mahoney et al. ${ }^{45}$ reported a larger prefrontal activation during a dual-task standing task in patients with Parkinson's disease compared with healthy subjects or patients with mild Parkinsonian signs. These findings also supported the notion that the PFC may compensate for the impaired postural ability in patients with neurological disorders. Together with previous findings from a behavioral study suggesting attentional demands for postural control, ${ }^{46}$ prefrontal involvement in postural task may be related to the attentional process in the maintenance of upright posture.

In contrast, the SMA and parietal association cortex were activated more with the preceding cues, suggesting that these cortices are involved in voluntary postural control. Recent studies with dynamic posturography suggested that the SMA is involved in volitional control of postural action and the parietal association cortex is involved in updating the spatial reference for postural control. ${ }^{47}$ In patients with hemiplegic stroke, we found that postural task-related cortical activation was reduced in the affected hemisphere, but there was a significant correlation between the individual postural ability and cortical activation in the SMA. ${ }^{48}$ We also investigated the longitudinal changes in balance-related cortical activation after intensive rehabilitation in patients with hemiplegic stroke. ${ }^{49}$ Group analysis of the longitudinal study revealed a significant facilitation of the SMA after rehabilitation, and the cortical activation changes in the SMA correlated significantly with balance recovery (Fig. 5). These findings also suggested that NIRS measurements would be useful as a surrogate marker of balance recovery after rehabilitation.

\section{Application of Near-Infrared Spectroscopy as a Therapeutic Tool in Neurorehabilitation}

\subsection{Near-Infrared Spectroscopy-Based Brain-Computer Interface}

One of the most exciting areas of clinical application of NIRS is the development of the BCI or brain-machine interface. BCI is the communication system bypassing the peripheral nervous system that allows the use of brain activity to control computers (a)

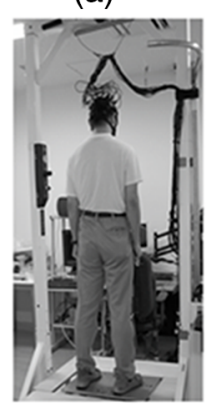

(b)

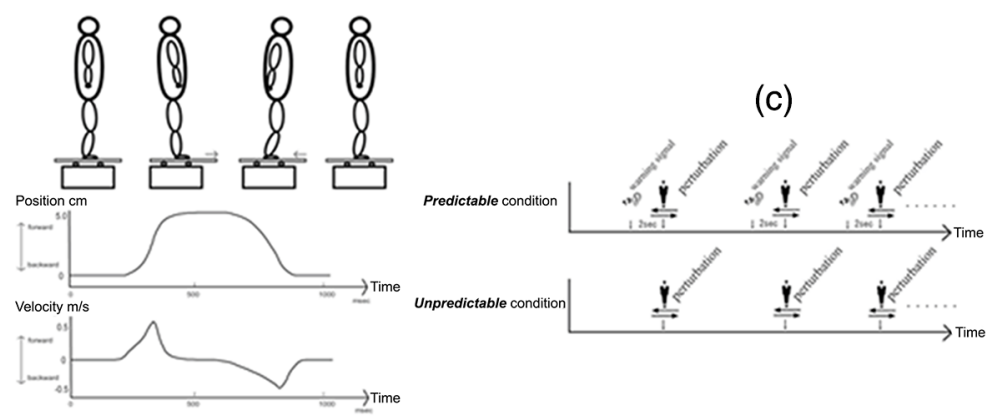

(d)

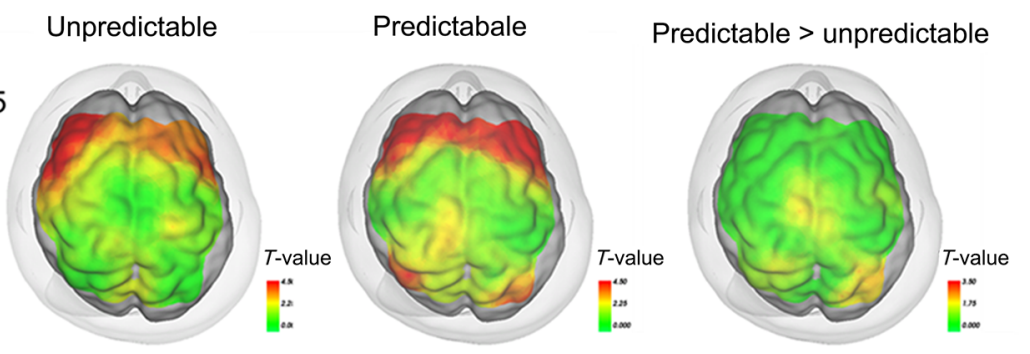

Fig. 4 Experimental setup for measurement of postural task-related cortical activation: (a) experimental overview, (b) schematic figure showing the platform movement, (c) task protocol in both conditions, and (d) cortical activation mapping based on the task-related OxyHb signal. Reproduced from the article by Mihara et al., ${ }^{43}$ where details are reported. () 2012 Masahito Mihara. Adapted from Applications of near infrared spectroscopy in neurorehabilitation. In Infrared Spectroscopy/Book 1 (ISBN: 979-953-307-3629); originally published under CC BY 3.0 license. Available from doi: 10.5772/2655. 
(a)

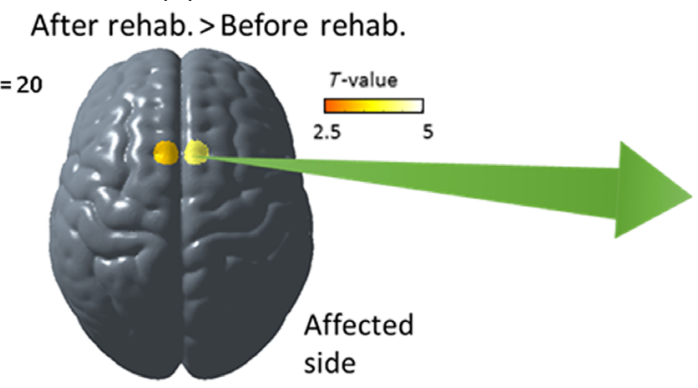

(b)

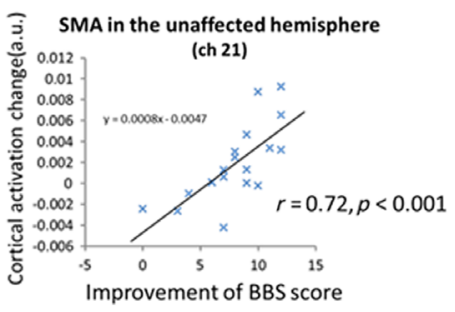

Fig. 5 Longitudinal changes of balance-related cortical activation: (a) the increased supplementary motor area activation after rehabilitation and (b) a significant correlation between cortical activation change and balance recovery. Reproduced from the article by Fujimoto et al., ${ }^{49}$ where details are reported. SMA, supplementary motor area and BBS, Berg balance scale.

or other devices. In severely affected patients, bypassing their impaired nervous system could be the only effective option to improve the ADL and to restore function. Therefore, one main purpose of the development of a BCI system is to substitute the lost function of the neuromuscular output. Among several techniques for detecting brain activity, NIRS has attracted attention because of its portable and noninvasive nature. Since NIRS signals contain physiological and experimental artifacts or errors, it is not straightforward to decode the acquired NIRS signal and several strategies have been proposed to improve decoding accuracy. Table 1 shows previous studies with NIRSmediated BCI system using motor imagery signals. For example, Sitaram et al. ${ }^{52}$ reported that right/left finger motor imagery could be classified with an average accuracy of $89 \%$ by applying the pattern recognition algorithm with a hidden Markov model to the multichannel NIRS data. Abdelnour and Huppert ${ }^{60}$ adopted an adaptive general linear model using Kalman filtering in the real-time analysis of NIRS. Although technical advances in signal decoding enable us to detect cortical activation associated with motor imagery with $70 \%$ to $90 \%$ of classification accuracy in healthy subjects (Table 1), patients with chronic severe paresis showed less cortical activation, especially in motor imagery, and classification accuracy tended to be lower in the motor imagery task in patients. ${ }^{61}$ In addition, since NIRS uses hemodynamic responses associated with neural activation, there were several seconds of delay between neural activation and NIRS signal changes. To overcome these disadvantages of NIRS-mediated BCI systems and to improve classification accuracy, a combined system with additional modalities such as the EEG-NIRS hybrid system has been introduced (Table 1). ${ }^{62}$

Despite improving the decoding techniques for NIRS signals, clinical usage of $\mathrm{BCI}$ in the rehabilitation field is limited

Table 1 NIRS-mediated $\mathrm{BCl}$ studies utilizing motor imagery signals.

\begin{tabular}{|c|c|c|c|}
\hline Reference & Brain area & Features & $\begin{array}{l}\text { Classification } \\
\text { accuracy (\%) }\end{array}$ \\
\hline \multicolumn{4}{|l|}{ NIRS-based system } \\
\hline Coyle et al. ${ }^{50}$ & Motor cortex & $\mathrm{HbO}$ value & 75 \\
\hline Coyle et al. ${ }^{51}$ & Motor cortex & $\mathrm{HbO}$ value & 70 to 90 \\
\hline Sitaram et al. ${ }^{52}$ & Bil motor cortex & Mean of $\Delta \mathrm{HbO}$ and $\mathrm{HbR}$ & 89.1 \\
\hline Holper and Wolf ${ }^{53}$ & Secondary motor cortex & $\mathrm{HbO}$ signals (mean, variance, skewness, and kurtosis) & 81 \\
\hline Naseer and Hong ${ }^{54}$ & Bil motor cortex & $\mathrm{HbO}$ signals (mean and slope of $\Delta \mathrm{HbO}$ ) & 87.2 \\
\hline Hong et al..$^{55}$ & Motor cortex and PFC & $\mathrm{HbO}$ signals (mean and slope of $\Delta \mathrm{HbO}$ ) & $\begin{array}{l}75.7 \text { (three class: } \\
\text { right/left/arithmetic) }\end{array}$ \\
\hline \multicolumn{4}{|c|}{ EEG-NIRS hybrid system } \\
\hline Fazli et al. ${ }^{56}$ & Bil motor cortex & NIRS: mean of $\Delta \mathrm{HbO}$ and $\mathrm{HbR}$ EEG: power & 83.1 \\
\hline Koo et al. ${ }^{57}$ & Bil motor cortex & NIRS: $\Delta \mathrm{HbO}$ EEG: power and variance & 88 (self-paced) \\
\hline Yin et al. ${ }^{58}$ & Bil motor cortex & $\begin{array}{l}\text { NIRS: signals from } \mathrm{HbR}, \mathrm{HbO}, \mathrm{HbT}, \mathrm{HbO}-\mathrm{HbR} \text {, light absorption } \\
\text { (two wavelengths) EEG: power, instantaneous phase, instantaneous } \\
\text { amplitude, and instantaneous frequency }\end{array}$ & 89 \\
\hline Buccino et al. ${ }^{59}$ & Bil motor cortex & NIRS: mean and slope of $\Delta \mathrm{HbO}$ and $\mathrm{HbR}$ EEG: power and variance & 72.2 (right/left) \\
\hline
\end{tabular}

Note: PFC, prefrontal cortex; $\mathrm{HbO}$, oxygenated hemoglobin; $\mathrm{HbR}$, deoxygenated hemoglobin; and $\mathrm{HbT}$, total hemoglobin. 
at this time. BCI can be used for restoration of multiple functions including communication and motor functions; however, the most rapidly developing usage of the BCI system is for communication. Recently, Gallegos-Ayala et al. ${ }^{63}$ reported that the NIRS-based BCI system showed an above chance level classification result in a patient with amyotrophic lateral sclerosis who was in a completely locked-in-state. These findings suggested that even in a locked-in-state patient, BCI enables the provision of a communication method.

\subsection{Near-Infrared Spectroscopy Application as a Neurofeedback Tool}

In addition to its use in a typical BCI system, NIRS could be used as a tool for augmenting brain plasticity. Recent advances in real-time signal processing technique enable the use of NIRS as a neurofeedback tool. In neurofeedback-based training, subjects can learn how to modulate cortical activity using real-time feedback of the target cortical activity to the subject.

According to this notion, we have developed an NIRS-mediated neurofeedback system (Fig. 6). ${ }^{48}$ We asked participants to undergo two neurofeedback trainings. The first condition was a motor imagery task with neurofeedback of contralateral motor cortex activity (real) and the other condition was motor imagery with an irrelevant random signal (sham). We found that motor imagery-related cortical activation was facilitated only in the real condition. Our data suggested that NIRS-based neurofeedback could be used as a feasible neuromodulation tool. Consistently, Kober et al. also reported the effect of NIRSmediated neurofeedback using motor imagery task on cortical

(a)

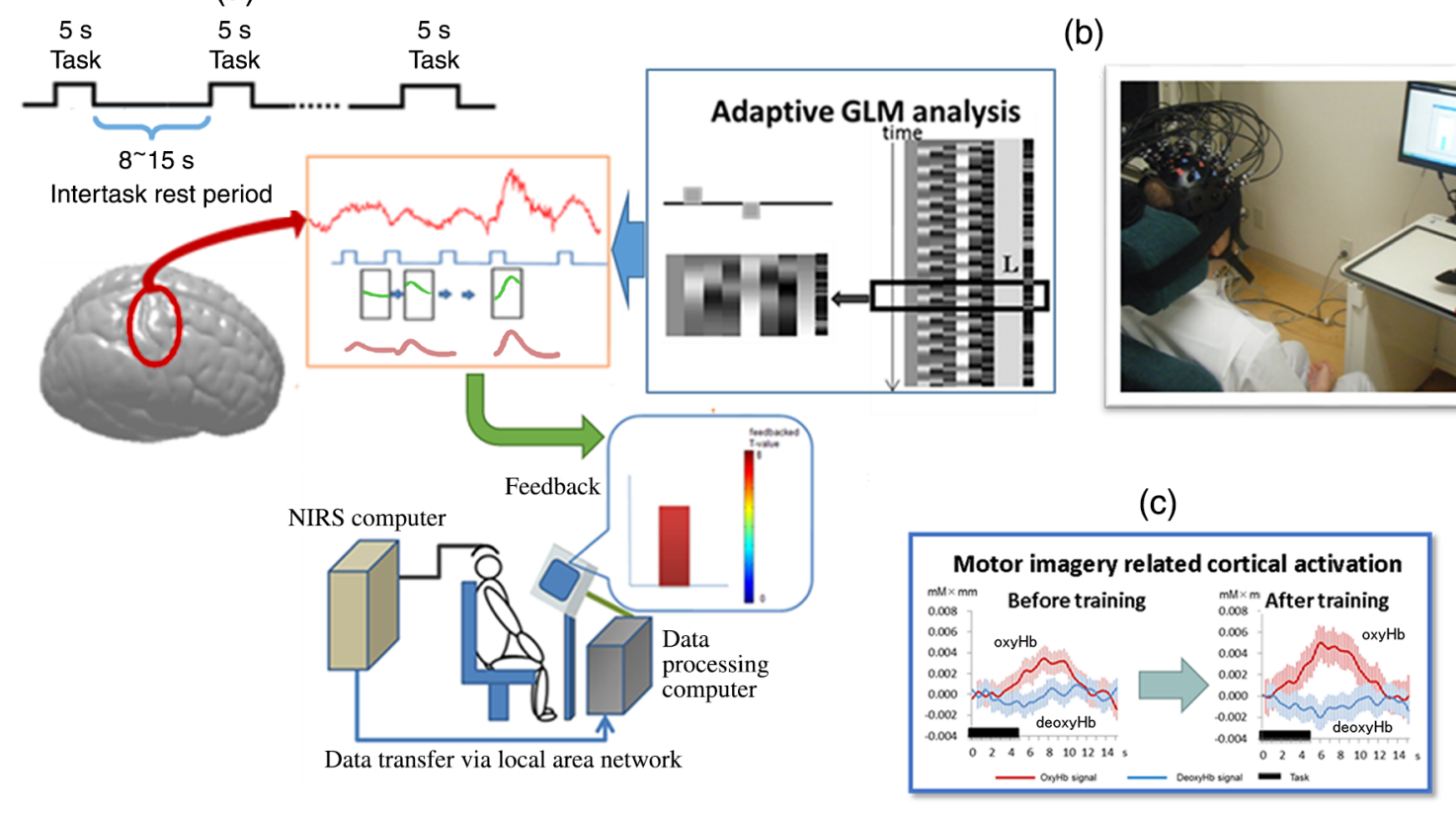

Fig. 6 (a) Schema of the NIRS-based neurofeedback system, (b) NIRS-based neurofeedback in use, and (c) cortical activity increased after neurofeedback. Reproduced from the article by Mihara et al., ${ }^{48}$ where details are reported. GLM: General Linear Model.
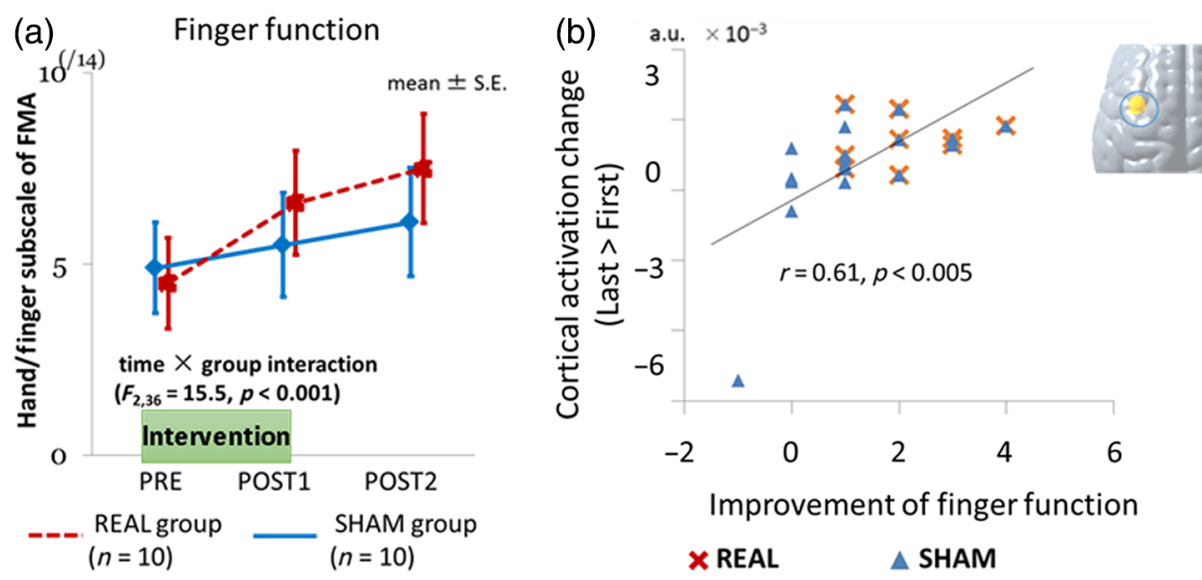

Fig. 7 NIRS-based neurofeedback for upper limb paresis in poststroke patients: (a) Neurofeedback improved upper limb function after stroke and (b) functional recovery correlated with cortical activation change. Reproduced from the article by Mihara et al., ${ }^{65}$ where details are reported. FMA: Fugl-Meyer Assessment. 
activation pattern. After eight sessions of neurofeedback training, only subjects with real neurofeedback showed localized cortical activation on the ipsilateral motor cortex during hand motor imagery, whereas subjects with sham feedback showed diffuse cortical activation pattern even after eight sessions of neurofeedback. ${ }^{64}$

We also investigated the therapeutic effect of this system for augmenting poststroke functional recovery in a small, randomized control trial. ${ }^{65}$ Twenty patients with subacute hemiplegic stroke were enrolled in this study. In addition to the usual intensive rehabilitation, patients participated in six sessions (three times/week) of neurofeedback intervention with motor imagery of paretic hand movement. Patients were randomly allocated to two groups: real and sham. As shown in Fig. 7, patients in the real group showed more improvement than patients in the sham group, with statistically significant interaction between groups and times $\left(F_{2,36}=15.5, p<0.001\right)$. Interestingly, motor imagery-related cortical activation changes significantly correlated with functional recovery after neurofeedback. Although further studies are needed to confirm the clinical efficacy, these findings suggested that NIRS-based neurofeedback could be used as a feasible therapeutic tool for poststroke patients.

\section{Conclusion}

NIRS is a unique neuroimaging tool with an inherent advantage of measuring cortical activation without onerous constraints, under daily-life circumstances. Because of these advantages, NIRS would be a suitable tool for investigating the neural underpinnings of dynamic motor tasks such as posture and gait control, proximal limb movement, or swallowing. However, it should be noted that physiological and experimental artifacts could easily contaminate NIRS signals. Nonetheless, further advances in signal processing techniques will expand the clinical usage of NIRS as a therapeutic tool.

\section{Acknowledgments}

We thank the "Research and Development Grants for Comprehensive Research for Persons with Disabilities" from the Japan Agency for Medical Research and Development (AMED), the "Development of Medical Devices and Systems for Advanced Medical Services" from AMED, the AMED-CREST from AMED, the Japan Foundation for Neuroscience and Mental Health, and the Osaka Medical Research Foundation for Intractable Diseases for funding support.

\section{References}

1. P. W. Duncan, S. M. Lai, and J. Keighley, "Defining post-stroke recovery: implications for design and interpretation of drug trials," Neuropharmacology 39(5), 835-841 (2000).

2. H. S. Jørgensen et al., "Stroke. Neurologic and functional recovery the copenhagen stroke study," Phys. Med. Rehabil. Clin. N. Am. 10(4), 887906 (1999).

3. C. Calautti and J. C. Baron, "Functional neuroimaging studies of motor recovery after stroke in adults: a review," Stroke 34(6), 1553-1566 (2003).

4. N. Ward and R. Frackowiak, "The cerebral basis of functional recovery," in Human Brain Function, R.S.J. Frackowiak et al., Eds., pp. 105-123, Academic Press, San Diego (2004).

5. J. J. Daly and J. R. Wolpaw, "Brain-computer interfaces in neurological rehabilitation," Lancet Neurol. 7(11), 1032-1043 (2008).

6. B. H. Dobkin, "Brain-computer interface technology as a tool to augment plasticity and outcomes for neurological rehabilitation," J. Physiol. 579(Pt. 3), 637-642 (2007).
7. N. Naseer and K.-S. Hong, "fNIRS-based brain-computer interfaces: a review," Front. Hum. Neurosci. 9, 3 (2015).

8. A. Delorme and S. Makeig, "EEG changes accompanying learned regulation of 12-Hz EEG activity," IEEE Trans. Neural Syst. Rehabil. Eng. 11(2), 133-137 (2003).

9. T. Fuchs et al., "Neurofeedback treatment for attention-deficit/hyperactivity disorder in children: a comparison with methylphenidate," Appl. Psychophysiol. Biofeedback 28(1), 1-12 (2003).

10. B. Kotchoubey et al., "Modification of slow cortical potentials in patients with refractory epilepsy: a controlled outcome study," Epilepsia 42(3), 406-416 (2001).

11. H. Johansen-Berg, "Functional imaging of stroke recovery: what have we learnt and where do we go from here?" Int. J. Stroke 2(1), 7-16 (2007).

12. N. S. Ward et al., "Neural correlates of motor recovery after stroke: a longitudinal fMRI study," Brain 126(Pt. 11), 2476-2496 (2003).

13. N. S. Ward et al., "Neural correlates of outcome after stroke: a crosssectional fMRI study," Brain 126(Pt. 6), 1430-1448 (2003).

14. H. Obrig and J. Steinbrink, "Non-invasive optical imaging of stroke," Philos. Trans. R. Soc. London, Ser. A 369(1955), 4470-4494 (2011).

15. G. Strangman et al., "Near-infrared spectroscopy and imaging for investigating stroke rehabilitation: test-retest reliability and review of the literature," Arch. Phys. Med. Rehabil. 87(12 Suppl. 2), S12-19 (2006).

16. H. Kato et al., "Near-infrared spectroscopic topography as a tool to monitor motor reorganization after hemiparetic stroke: a comparison with functional MRI,' Stroke 33(8), 2032-2036 (2002).

17. S.-W. Park et al., "Changes in serial optical topography and TMS during task performance after constraint-induced movement therapy in stroke: a case study," Neurorehabil. Neural Repair 18(2), 95-105 (2004).

18. K. Takeda et al., "Shift of motor activation areas during recovery from hemiparesis after cerebral infarction: a longitudinal study with nearinfrared spectroscopy," Neurosci. Res. 59(2), 136-144 (2007).

19. J. Cao et al., "Evaluation of cortical plasticity in children with cerebral palsy undergoing constraint-induced movement therapy based on functional near-infrared spectroscopy," J. Biomed. Opt. 20(4), 046009 (2015).

20. D. R. Leff et al., "Changes in prefrontal cortical behaviour depend upon familiarity on a bimanual co-ordination task: an fNIRS study," Neurolmage 39(2), 805-813 (2008).

21. M. Hatakenaka et al., "Frontal regions involved in learning of motor skill-a functional NIRS study," NeuroImage 34(1), 109-116 (2007).

22. M. Hatakenaka et al., "Impaired motor learning by a pursuit rotor test reduces functional outcomes during rehabilitation of poststroke ataxia," Neurorehabil. Neural Repair 26(3), 293-300 (2012).

23. S. T. Grafton et al., "Functional anatomy of human procedural learning determined with regional cerebral blood flow and PET," J. Neurosci. 12(7), 2542-2548 (1992).

24. J. L. O'Loughlin et al., "Incidence of and risk factors for falls and injurious falls among the community-dwelling elderly," Am. J. Epidemiol. 137(3), 342-354 (1993).

25. D. M. Armstrong, "The supraspinal control of mammalian locomotion," J. Physiol. 405, 1-37 (1988).

26. T. Drew, S. Prentice, and B. Schepens, "Cortical and brainstem control of locomotion," Prog. Brain Res. 143, 251-261 (2004).

27. V. Dietz, J. Quintern, and W. Berger, "Cerebral evoked potentials associated with the compensatory reactions following stance and gait perturbation," Neurosci. Lett. 50(1-3), 181-186 (1984).

28. S. Quant, B. E. Maki, and W. E. McIlroy, "The association between later cortical potentials and later phases of postural reactions evoked by perturbations to upright stance," Neurosci. Lett. 381(3), 269-274 (2005).

29. S. Slobounov et al., "Role of cerebral cortex in human postural control: an EEG study," Clin. Neurophysiol. 116(2), 315-323 (2005).

30. I. Miyai et al., "Cortical mapping of gait in humans: a near-infrared spectroscopic topography study," NeuroImage 14(5), 1186-1192 (2001).

31. M. Suzuki et al., "Prefrontal and premotor cortices are involved in adapting walking and running speed on the treadmill: an optical imaging study," Neurolmage 23(3), 1020-1026 (2004).

32. R. Holtzer et al., "Online fronto-cortical control of simple and attentiondemanding locomotion in humans," NeuroImage 112, 152-159 (2015).

33. E. Al-Yahya et al., "Prefrontal cortex activation while walking under dual-task conditions in stroke: a multimodal imaging study," Neurorehabil. Neural Repair 30(6), 591-599 (2016). 
34. R. Holtzer et al., "Neurological gait abnormalities moderate the functional brain signature of the posture first hypothesis," Brain Topogr. 29(2), 334-343 (2016).

35. I. Miyai et al., "Premotor cortex is involved in restoration of gait in stroke," Ann. Neurol. 52(2), 188-194 (2002).

36. S. Sangani, A. Lamontagne, and J. Fung, "Cortical mechanisms underlying sensorimotor enhancement promoted by walking with haptic inputs in a virtual environment," Prog. Brain Res. 218, 313-330 (2015).

37. I. Miyai et al., "Longitudinal optical imaging study for locomotor recovery after stroke," Stroke 34(12), 2866-2870 (2003).

38. I. Miyai et al., "Middle cerebral artery stroke that includes the premotor cortex reduces mobility outcome," Stroke 30(7), 1380-1383 (1999).

39. P.-Y. Lin, J.-J. Chen, and S.-I. Lin, "The cortical control of cycling exercise in stroke patients: an fNIRS study," Hum. Brain Mapp. 34(10), 2381-2390 (2013).

40. M. Mihara et al., "Sustained prefrontal activation during ataxic gait: a compensatory mechanism for ataxic stroke?" NeuroImage 37(4), 13381345 (2007).

41. P. Caliandro et al., "Prefrontal cortex controls human balance during overground ataxic gait," Restor. Neurol. Neurosci. 30(5), 397-405 (2012).

42. P. Caliandro et al., "Prefrontal cortex as a compensatory network in ataxic gait: a correlation study between cortical activity and gait parameters," Restor. Neurol. Neurosci. 33(2), 177-187 (2015).

43. M. Mihara et al., "Role of the prefrontal cortex in human balance control," Neurolmage 43(2), 329-336 (2008).

44. M. Ferrari et al., "Prefrontal cortex activated bilaterally by a tilt board balance task: a functional near-infrared spectroscopy study in a semiimmersive virtual reality environment," Brain Topogr. 27(3), 353-365 (2014).

45. J. R. Mahoney et al., "The role of prefrontal cortex during postural control in Parkinsonian syndromes a functional near-infrared spectroscopy study," Brain Res. 1633, 126-138 (2016).

46. M. Woollacott and A. Shumway-Cook, "Attention and the control of posture and gait: a review of an emerging area of research," Gait Posture 16(1), 1-14 (2002).

47. H. Takakura et al., "Cerebral hemodynamic responses during dynamic posturography: analysis with a multichannel near-infrared spectroscopy system," Front. Hum. Neurosci. 9, 620 (2015).

48. M. Mihara et al., "Neurofeedback using real-time near-infrared spectroscopy enhances motor imagery related cortical activation," PLoS One 7(3), e32234 (2012).

49. H. Fujimoto et al., "Cortical changes underlying balance recovery in patients with hemiplegic stroke," NeuroImage 85(Pt. 1), 547-554 (2014).

50. S. Coyle et al., "On the suitability of near-infrared (NIR) systems for next-generation brain-computer interfaces," Physiol. Meas. 25(4), 815822 (2004).

51. S. M. Coyle, T. E. Ward, and C. M. Markham, "Brain-computer interface using a simplified functional near-infrared spectroscopy system," J. Neural Eng. 4(3), 219-226 (2007).

52. R. Sitaram et al., "Temporal classification of multichannel near-infrared spectroscopy signals of motor imagery for developing a brain-computer interface," NeuroImage 34(4), 1416-1427 (2007).
53. L. Holper and M. Wolf, "Single-trial classification of motor imagery differing in task complexity: a functional near-infrared spectroscopy study," J. Neuroeng. Rehabil. 8, 34 (2011).

54. N. Naseer and K.-S. Hong, "Classification of functional near-infrared spectroscopy signals corresponding to the right- and left-wrist motor imagery for development of a brain-computer interface," Neurosci. Lett. 553, 84-89 (2013).

55. K.-S. Hong, N. Naseer, and Y.-H. Kim, "Classification of prefrontal and motor cortex signals for three-class fNIRS-BCI," Neurosci. Lett. 587, 87-92 (2015).

56. S. Fazli et al., "Enhanced performance by a hybrid NIRS-EEG brain computer interface," NeuroImage 59(1), 519-529 (2012).

57. B. Koo et al., "A hybrid NIRS-EEG system for self-paced brain computer interface with online motor imagery," J. Neurosci. Methods 244, 26-32 (2015).

58. X. Yin et al., "A hybrid BCI based on EEG and fNIRS signals improves the performance of decoding motor imagery of both force and speed of hand clenching," J. Neural Eng. 12(3), 036004 (2015).

59. A. P. Buccino, H. O. Keles, and A. Omurtag, "Hybrid EEG-fNIRS asynchronous brain-computer interface for multiple motor tasks," PLoS One 11(1), e0146610 (2016).

60. A. F. Abdelnour and T. Huppert, "Real-time imaging of human brain function by near-infrared spectroscopy using an adaptive general linear model," Neurolmage 46(1), 133-143 (2009).

61. Y. Blokland et al., "Combined EEG-fNIRS decoding of motor attempt and imagery for brain switch control: an offline study in patients with tetraplegia," IEEE Trans. Neural Syst. Rehabil. Eng. 22(2), 222-229 (2014).

62. G. Pfurtscheller et al., "The hybrid BCI," Front. Neurosci. 4, 30 (2010).

63. G. Gallegos-Ayala et al., "Brain communication in a completely lockedin patient using bedside near-infrared spectroscopy," Neurology 82(21), 1930-1932 (2014).

64. S. E. Kober et al., "Near-infrared spectroscopy based neurofeedback training increases specific motor imagery related cortical activation compared to sham feedback," Biol. Psychol. 95, 21-30 (2014).

65. M. Mihara et al., "Near-infrared spectroscopy-mediated neurofeedback enhances efficacy of motor imagery-based training in poststroke victims: a pilot study," Stroke 44(4), 1091-1098 (2013).

Masahito Mihara received his PhD in neuroscience from Osaka University in 2007 . He is an associate professor at Osaka University. His current research interests include the development of a therapeutic system using near-infrared spectroscopy (NIRS)-mediated neurofeedback and investigating neural mechanisms of human motor control in both healthy and neurological patients using multimodal neuroimaging technique including NIRS.

Ichiro Miyai is the medical director at Morinomiya Hospital and vice president of the Omichikai Medical Group. He is an associate editor of the Neurorehabilitation and Neural Repair journal. His current research interests include neural mechanisms of functional repair after brain damage and the development of a therapeutic system using NIRS-mediated neurofeedback. 